\title{
ACOPLAMENTO DAS REAÇÕES DE DESIDRATAÇÃO DE ETANOL E DE COMBUSTÃO CATALÍTICA DE METANO EM REATOR MULTIFUNCIONAL
}

\author{
L. F. NOVAZZI ${ }^{1}$ e G. J. M. FORMON ${ }^{1}$ \\ ${ }^{1}$ Centro Universitário da FEI, Departamento de Engenharia Química \\ E-mail para contato: lnovazzi@fei.edu.br
}

\begin{abstract}
RESUMO - Reatores multifuncionais são aqueles nos quais há acoplamento de reações exotérmicas com reações endotérmicas, de modo que as primeiras forneçam calor para essas últimas. Isso é bastante interessante, pois num único equipamento são combinadas mais de duas operações unitárias. Nesse projeto estudou-se, através de simulação, a reação de desidratação catalítica do etanol, de caráter endotérmico e na qual se produz etileno, combinada com a combustão de metano, num reator multifuncional. Foi feita a modelagem matemática do reator, escrevendo-se as equações de balanço molar para cada um dos componentes do sistema reacional, juntamente com as equações de conservação de energia para as reações exotérmicas e para as endotérmicas. As cinéticas dessas reações foram levantadas em literatura. A modelagem foi feita em regime permanente, por meio de equações diferenciais ordinárias, que foram resolvidas em Matlab. Foram estudadas configurações de escoamento em paralelo e em contracorrente no reator e os resultados obtidos mostraram comportamento semelhante para conversão e rendimento das reações. Nas melhores condições, a conversão da reação de desidratação do etanol ficou na faixa de $85 \%$ a $90 \%$, com rendimento em etileno entre $90 \%$ e $95 \%$.
\end{abstract}

\section{INTRODUÇÃO}

Nas indústrias químicas e petroquímicas é fabricada uma grande gama de produtos essenciais para a sociedade moderna, tais como plásticos, fertilizantes e defensivos agrícolas, tintas e vernizes, sabões e detergentes, fármacos, produtos de higiene, cosméticos, etc. Existe considerável pressão sobre essas indústrias em relação à sua sustentabilidade e, nesse aspecto, uma das tendências que têm surgido nos últimos anos na área de Engenharia Química é a chamada Intensificação de Processos ou PI (Lutze et al., 2010).

Uma das formas de se fazer a PI é através da integração de duas ou mais operações unitárias, possibilitando que a planta opere com equipamentos de tamanho reduzido. A integração ou acoplamento de reações exotérmicas com reações endotérmicas em reatores multifuncionais é uma maneira interessante de se promover a PI, desde que a temperatura das reações exotérmicas seja maior do que aquela das reações endotérmicas (Rahimpour et al., 2012).

O acoplamento de reações exotérmicas e endotérmicas pode ser realizado de três diferentes 


\section{9 a 22 de outubro de 2014 \\ Florianópolis/SC}

modos: direto, regenerativo ou recuperativo. No acoplamento recuperativo as reações são separadas por uma membrana ou por uma parede metálica, que permitem a troca de calor. Na Figura 1 é esquematizado um reator com acoplamento recuperativo, sem membrana, em duas configurações de escoamento: contracorrente (Figura 1a) e paralelo (Figura 1b).

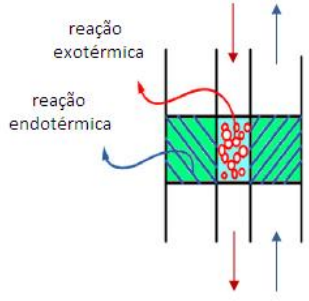

(a) contracorrente

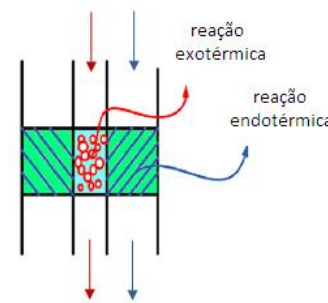

(b) paralelo

Figura 1 - Esquema de reator com acoplamento recuperativo.

$\mathrm{Na}$ figura anterior, as setas vermelhas e azuis representam as correntes nas quais ocorrem as reações de natureza exotérmica e endotérmica, respectivamente. Existem estudos de reatores multifuncionais que consistem num trocador de calor a placas, com o catalisador colocado entre elas. Esses equipamentos recebem o nome de reatores catalíticos a placas. No trabalho de Zanfir e Gavriilidis (2001), por exemplo, fez-se a modelagem de um reator catalítico a placas envolvendo a desidrogenação de etano, produzindo etileno, acoplada com a combustão de metano.

Contudo, uma das formas mais simples de reator com acoplamento recuperativo consiste num trocador de calor casco e tubo. As partículas de catalisador para a reação exotérmica são colocadas no interior dos tubos, enquanto que as partículas de catalisador para a reação endotérmica são colocadas na região do casco. Na Figura 1 essas partículas são respectivamente indicadas em vermelho e por uma área hachurada em azul e verde.

Diversos trabalhos na literatura lidaram com o acoplamento de reações exotérmicas e endotérmicas, como se pode observar na extensa revisão feita por Rahimpour et al (2012). Dentre os acoplamentos recuperativos sem membrana estudados e com aplicação industrial mais importante, pode-se citar a associação da combustão de metano com a desidrogenação de propano a propileno (Annaland et al., 2002) e o acoplamento da combustão do metano com a reação de reforma do metano com vapor de água (Kolios et al., 2002).

O etileno é uma matéria-prima importante na indústria petroquímica, sendo utilizado na produção de óxido de etileno e de diversos polímeros, tais como polietileno (PE), polietileno tereftalato (PET) e policloreto de vinila (PVC). Essa matéria-prima é tradicionalmente obtida a partir do processamento de frações do petróleo. Entretanto, em virtude do aumento de preços de óleo no mercado internacional, a produção de etileno a partir da desidratação catalítica de etanol, que é renovável, começou a se tornar uma rota atrativa. Essa reação de desidratação, de caráter bastante endotérmico, é normalmente conduzida em fase gasosa, num leito catalítico de alumina, em temperaturas entre $240^{\circ} \mathrm{C}$ e $450{ }^{\circ} \mathrm{C}$ (Kagyrmanova et al., 2011). Essa rota possui particular interesse para o Brasil, que é um dos maiores produtores mundiais de etanol. 
Podem ser encontrados na literatura trabalhos que abordaram a cinética da desidratação catalítica de etanol (Aquino, 1989; Lima, 2010; Kagyrmanova et al., 2011). Contudo, na pesquisa bibliográfica realizada, não se achou nenhum trabalho que lidou com o acoplamento da reação de desidratação do etanol com reações exotérmicas, num reator multifuncional. Como o nível de temperatura para realizar essa desidratação é relativamente elevado, a escolha das reações exotérmicas fica mais restrita, já que deve haver força motriz para que a corrente quente, na qual ocorrem as reações exotérmicas, transfira calor para a corrente mais fria, na qual acontecem as reações endotérmicas. Uma das possibilidades é a combustão catalítica do metano, num leito de platina / paládio e que ocorre em temperaturas próximas a $550{ }^{\circ} \mathrm{C}$ (Abbasi et al., 2012). No contexto apresentado, o objetivo desse trabalho é estudar, através de simulação, o acoplamento recuperativo da reação de desidratação do etanol, de natureza endotérmica, com a reação de combustão catalítica do metano, de característica exotérmica, num reator multifuncional contínuo, de leito fixo tanto no lado exotérmico como no endotérmico.

\section{METODOLOGIA}

A produção de etileno a partir da desidratação do etanol (Equação 1), catalisada por alumina em reator heterogêneo, envolve diversas reações secundárias (Equações 2 a 5). Assim, as Equações 1 a 5 representam as reações que fazem parte do sistema reacional endotérmico, totalizando 7 diferentes componentes. Pode-se observar que os subprodutos desse sistema são dietil éter (Equação 2), acetaldeído (Equação 4) e butileno (Equação 5). A cinética dessas reações está disponível em literatura (Kagyrmanova et al., 2011).

$$
\begin{aligned}
& \mathrm{C}_{2} \mathrm{H}_{5} \mathrm{OH} \rightarrow \mathrm{C}_{2} \mathrm{H}_{4}+\mathrm{H}_{2} \mathrm{O} \\
& 2 \mathrm{C}_{2} \mathrm{H}_{5} \mathrm{OH} \rightarrow\left(\mathrm{C}_{2} \mathrm{H}_{5}\right)_{2} \mathrm{O}+\mathrm{H}_{2} \mathrm{O} \\
& \left(\mathrm{C}_{2} \mathrm{H}_{5}\right)_{2} \mathrm{O} \rightarrow 2 \mathrm{C}_{2} \mathrm{H}_{4}+\mathrm{H}_{2} \mathrm{O} \\
& \mathrm{C}_{2} \mathrm{H}_{5} \mathrm{OH} \rightarrow \mathrm{C}_{2} \mathrm{H}_{4} \mathrm{O}+\mathrm{H}_{2} \\
& 2 \mathrm{C}_{2} \mathrm{H}_{4} \rightarrow \mathrm{C}_{4} \mathrm{H}_{8}
\end{aligned}
$$

Já a reação exotérmica, de combustão catalítica do metano, é apresentada na Equação 6 e sua cinética também pode ser encontrada em literatura (Abbasi et al., 2012). Fazem parte desse meio reacional exotérmico 5 componentes: o metano, o oxigênio, o gás carbônico, a água e também o nitrogênio, que é usado como diluente.

$$
\mathrm{CH}_{4}+2 \mathrm{O}_{2} \rightarrow \mathrm{CO}_{2}+2 \mathrm{H}_{2} \mathrm{O}
$$


Para a modelagem realizada nesse trabalho, foram consideradas as seguintes hipóteses:

- Comportamento de gás ideal da mistura;

- Variações radiais de temperatura e de concentração desprezadas;

o Resistência à transferência de massa desprezível no interior do catalisador;

o Troca de calor entre as reações exotérmicas e endotérmicas proporcional a um coeficiente global de transferência de calor $U$, considerado constante;

- Regime permanente;

o Sem troca de calor com o ambiente.

A modelagem fenomenológica foi realizada com base no balanço molar (Equação 7), que deve ser escrito para cada um dos $j$ componentes que fazem parte do sistema reacional endotérmico (desidratação do etanol) e exotérmico (combustão do metano). Na Equação $7, F_{j}$ e $r_{i j}$ representam, respectivamente, a vazão molar e a velocidade de reação do componente $j, W$ é a massa de catalisador da reação endotérmica e $q$ é o número de reações que estão ocorrendo (Fogler, 2009). Para os sistemas reacionais endotérmico e exotérmico, $q$ assumirá os valores de 5 e 1 , respectivamente.

$$
\frac{d F_{j}}{d W}=\sum_{i=1}^{q} r_{i j}
$$

A velocidade de reação $r_{i}$ para as reações envolvidas na desidratação do etanol são descritas na Equação 8, na qual $A_{i}$ e $E_{i}$ são, respectivamente, a constante pré-exponencial e a energia de ativação da reação $i ; R$ é a constante universal dos gases ideais; $T$ é a temperatura da corrente na qual a reação ocorre; $P_{j}$ é a pressão parcial do reagente $j$ e $n$ é a ordem da reação $i$.

$$
r_{i}=A_{i} e^{\left(-E_{i} / R T\right)} P_{j}^{n}
$$

Os valores das constantes pré-exponenciais $A_{i}$, das energias de ativação $E_{i}$, das ordens $n$ e as entalpias padrões a $25^{\circ} \mathrm{C}, \Delta H_{r}^{o}$, para cada uma das 5 reações envolvidas (Equações 1 a 5 ) na desidratação do etanol são apresentadas na Tabela 1. Já a velocidade de combustão catalítica do metano é escrita em função de sua concentração molar $C_{\text {metano }}$. Para essa reação, considerada de primeira ordem, a constante pré-exponencial é igual a $1,79 \cdot 10^{6} \mathrm{~s}^{-1}$ e a energia de ativação vale 81,0 $\mathrm{kJ} \cdot \mathrm{mol}^{-1}$ (Abbasi et al., 2012). A entalpia dessa reação de combustão a $25^{\circ} \mathrm{C}$ é de $-802,620 \mathrm{~kJ} \cdot \mathrm{mol}^{-1}$.

Tabela 1 - Parâmetros cinéticos e termodinâmicos das reações de desidratação do etanol

\begin{tabular}{ccccc}
\hline Reação & $\begin{array}{c}A_{i} / \\
\left(\mathrm{mol} \cdot \mathrm{atm}^{-n} \cdot \mathrm{kg}^{-1} \cdot \mathrm{s}^{-1}\right)\end{array}$ & $E_{i} /\left(\mathrm{kJ} \cdot \mathrm{mol}^{-1}\right)$ & $n$ & $\Delta H_{r}^{o} /\left(\mathrm{kJ} \cdot \mathrm{mol}^{-1}\right)$ \\
\hline 1 & $5,41 \cdot 10^{6}$ & 147,7 & 1 & 45,646 \\
2 & $9,55 \cdot 10^{7}$ & 101,0 & 2 & $-12,007$ \\
3 & $2,79 \cdot 10^{10}$ & 135,0 & 1 & 115,306 \\
4 & $2,78 \cdot 10^{8}$ & 138,4 & 1 & 68,550 \\
5 & $1,45 \cdot 10^{7}$ & 113,7 & 2 & 55,660 \\
\hline
\end{tabular}

Fonte: Kagyrmanova et al., 2011 
Além das equações de balanço molar para cada um dos $j$ componentes, devem ser acrescentados mais dois balanços de energia, um para as reações exotérmicas, de temperatura $T_{\text {exo }}$, e um para as reações endotérmicas, de temperatura $T_{\text {endo }}$. Esses balanços são expressos nas Equações 9 e 10 , respectivamente, nas quais $U$ representa o coeficiente global de transferência de calor, $\rho_{b}$ é a densidade aparente do leito, $D$ é o diâmetro do tubo, $\Delta H_{r, i}$ é a entalpia da i-ésima reação, $C_{P, j}$ é o calor específico do componente $j$ e $m$ é o número total de componentes do meio reacional. Para os meios endotérmico e exotérmico, $m$ será igual a 7 e 5 , respectivamente.

$$
\begin{aligned}
& \frac{d T_{\text {endo }}}{d W}=\left(\frac{1}{\sum_{j=1}^{m} F_{j} C_{P, j}}\right)\left[\frac{4 U}{\rho_{b, \text { endo }} D}\left(T_{\text {exo }}-T_{\text {endo }}\right)+\sum_{i=1}^{q} r_{i j} \Delta H_{r, i}\right] \\
& \frac{d T_{\text {exo }}}{d W}=\left(\frac{1}{\sum_{j=1}^{m} F_{j} C_{P, j}}\right)\left[\frac{4 U}{\rho_{\text {bexo }} D}\left(T_{\text {endo }}-T_{\text {exo }}\right)+\sum_{i=1}^{q} r_{i j} \Delta H_{r, i}\right]
\end{aligned}
$$

Também foi considerada a perda de carga nos dois leitos catalíticos, por meio da equação de Ergun. Do ponto de vista matemático, a modelagem proposta nesse trabalho é caracterizada por ser um problema de valor inicial, constituído por um sistema de equações diferenciais ordinárias: 12 equações provenientes dos balanços molares (Equação 7), 2 oriundas dos balanços de energia e 2 resultantes de perda de carga. Esse sistema de equações diferenciais foi resolvido em Matlab, através da função ode45.

Foram calculados o grau de conversão do etanol e o rendimento em etileno, sendo esse último definido como o etileno produzido em relação ao etanol convertido. Nas simulações realizadas, considerou-se para a reação endotérmica uma alimentação de etanol puro ao reator e para a reação exotérmica uma relação de alimentação de metano / oxigênio de 1 para 8, juntamente com nitrogênio inerte. A pressão de alimentação $P_{0}$, as faixas de temperatura de alimentação $T_{0}$ e o tempo espacial $\tau_{o}$ para as reações são especificados na Tabela 2. Para o coeficiente global de transferência de calor $U$ foi considerado um valor de $100 \mathrm{~W} \cdot \mathrm{m}^{-2} \cdot \mathrm{K}^{-1}$.

Tabela 2 - Condições de alimentação

\begin{tabular}{cccc}
\hline Reação & $P_{0} / \mathrm{kPa}$ & $T_{0} / \mathrm{K}$ & $\tau_{o} / \mathrm{s}$ \\
\hline Endotérmica & 200 & 550 a 750 & 0,14 \\
Exotérmica & 250 & 750 a 950 & 1,67 \\
\hline
\end{tabular}

\section{RESULTADOS E DISCUSSÃO}

A partir das condições descritas no parágrafo anterior e na Tabela 2, fez-se um mapeamento da influência da temperatura de alimentação das correntes sobre o grau de conversão do etanol e o rendimento em etileno, com escoamento paralelo, como indicado na Figura 2. Observando-se essa figura, nota-se que o grau de conversão do etanol varia entre $55 \%$ a $82 \%$, enquanto que o rendimento em etileno varia de $30 \%$ a $90 \%$. Além disso, percebe-se que maiores temperaturas de alimentação das correntes nessas reações acopladas favorecem tanto o grau de conversão quanto o rendimento. Essas regiões analisadas foram determinadas tendo-se como referência o tempo espacial $\tau_{o}$ de $0,14 \mathrm{~s}$ para a 
reação endotérmica dentro do reator multifuncional.
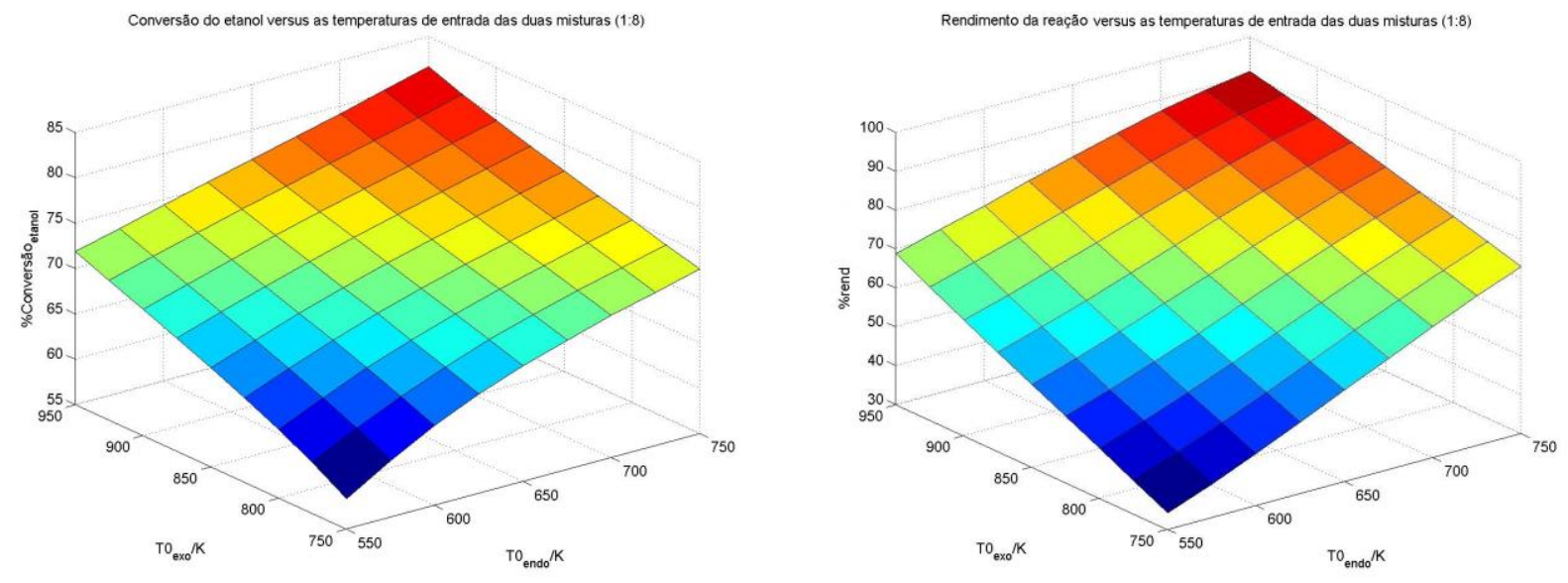

Figura 2 - Mapeamento do grau de conversão e rendimento para a reação endotérmica.

Contudo, o próprio tempo espacial é outra variável que pode ser estudada nesse sistema. Tomando-se como exemplo temperaturas de alimentação para as reações endotérmica e exotérmica de $650 \mathrm{~K}$ e $900 \mathrm{~K}$, respectivamente, a seguir são apresentados os perfis adimensionais de vazão (Figura 3a) e o grau de conversão do etanol e o rendimento em etileno para a reação endotérmica (Figura 3b), em função do tempo espacial $\tau_{o}$ e com escoamento paralelo. As vazões adimensionais são escritas como a relação entre a vazão do componente e a vazão do reagente limitante na entrada do reator.

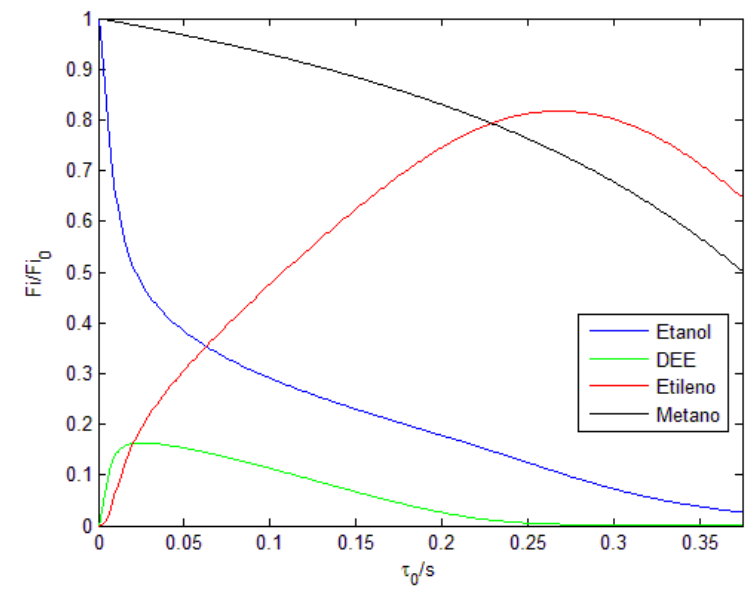

(a) Perfis de vazão adimensional

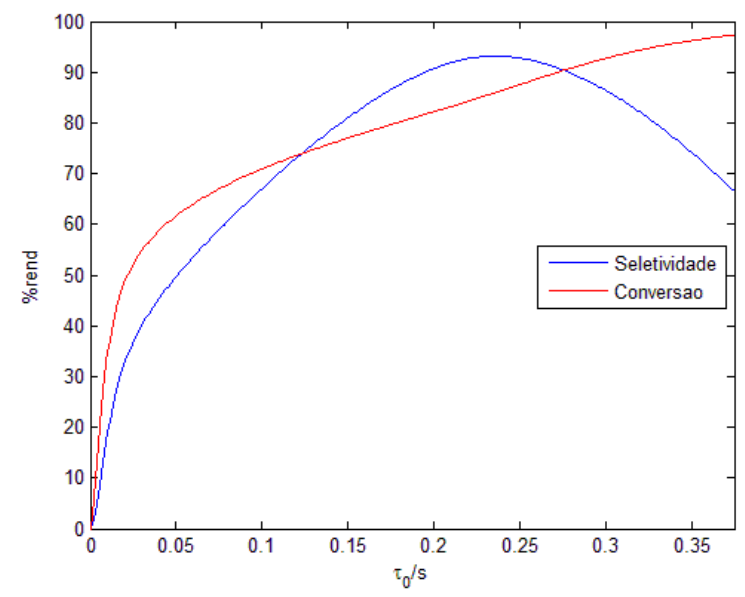

(b) Conversão e rendimento

Figura 3 - Influência do tempo espacial sobre as vazões, conversão e rendimento.

Os resultados da Figura 3 indicam que logo na entrada do reator, com tempo espacial de 0,01 s, cerca de metade do etanol é consumido, produzindo principalmente dietil éter (DEE). O éter obtido é posteriormente convertido em etileno (Equação 3), conforme se percebe pela análise do perfil de 
vazão adimensional de DEE e de etileno (Figura 3a). O ponto de máximo rendimento da reação, de 93,2\%, ocorre num tempo espacial de 0,235 s, com conversão de 85,9\% (Figura 3b). Nesse ponto, a conversão da reação exotérmica, que corresponde à queima catalítica do metano, foi de 21,5\%, conforme se pode calcular pelo perfil de vazão de metano na Figura 3a.

Nas Figuras 4a e 4b são apresentados os perfis de temperatura para as reações endotérmica e exotérmica, respectivamente, em função do tempo espacial $\tau_{o}$, com temperaturas de alimentação $T_{0, \text { endo }}$ e $T_{0, \text { exo }}$ de $650 \mathrm{~K}$ e $900 \mathrm{~K}$. Nessas figuras, os perfis em azul representam escoamento em paralelo e aqueles em rosa indicam escoamento em contracorrente. Analisando-se o perfil de temperaturas da reação endotérmica, vê-se que na entrada do reator há um aumento de temperatura, por conta da troca de calor com a corrente quente e por conta da velocidade das reações endotérmicas ainda serem pequenas, com pouco calor sendo absorvido. Em seguida, quando a temperatura da corrente fria atinge um pico, numa temperatura próxima a $680 \mathrm{~K}$, aumenta a energia absorvida pelas reações endotérmicas e o calor trocado entre as correntes não é mais capaz de sustentar essa demanda energética, fazendo com que a temperatura caia. Os perfis de temperatura da reação endotérmica são muito semelhantes tanto para o escoamento em paralelo como para aquele em contracorrente. Desse modo, a conversão e o rendimento máximos da reação endotérmica foram praticamente iguais para o escoamento paralelo e o contracorrente. A única diferença entre esses pontos de máximo corresponde ao tempo espacial: no caso de escoamento paralelo, esse tempo espacial foi de 0,235 s e no caso de escoamento contracorrente foi de $0,223 \mathrm{~s}$.

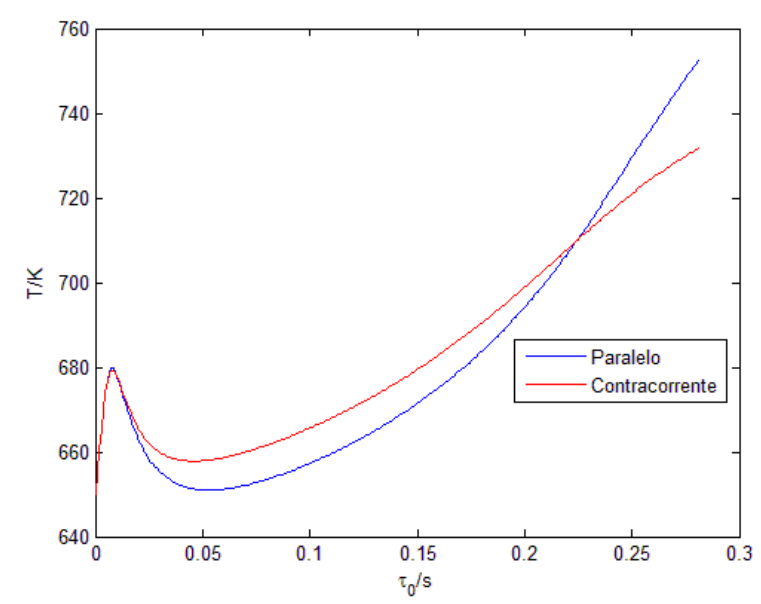

(a) endotérmica

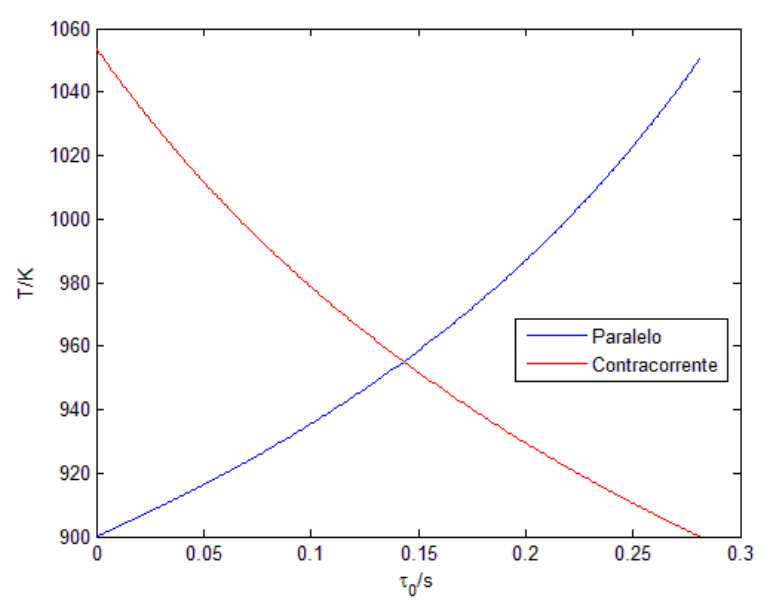

(b) exotérmica

Figura 4 - Perfis de temperatura para escoamento em paralelo e em contracorrente.

\section{CONCLUSÕES}

Os resultados obtidos na simulação do acoplamento das reações de desidratação de etanol e de combustão catalítica de metano, em reator multifuncional, mostraram que é interessante se ter uma temperatura elevada das correntes na alimentação do reator, a fim de se aumentar o grau de conversão da reação de desidratação e também o rendimento em etileno no processo (Figura 2). Verificou-se também nesse trabalho que, além das temperaturas das correntes de alimentação, o tempo espacial é 
bastante importante para se ter bom rendimento em etileno (Figura 3b). Nas melhores condições estudadas, conseguiu-se um grau de conversão do etanol de mais de $85 \%$ e um rendimento em etileno de mais de 93\%. Mostrou-se que nesse sistema reacional o fato de se ter escoamento paralelo ou contracorrente leva a perfis de temperatura muito semelhantes entre si (Figura 4), com graus de conversão e rendimentos também próximos.

\section{REFERÊNCIAS}

ABBASI, R.; WU, L.; WANKE, S.E.; HAYES, R.E. Kinetics of methane combustion over Pt and Pt-Pd catalysts. Chemical Engineering Research and Design, v.90, p.1930-1942, 2012.

ANNALAND, M.V.S.; NIJSSEN, R.C. A novel reverse flow reactor coupling endothermic and exothermic reactions: an experimental study. Chemical Engineering Science, v.57, p.4967-4985, 2002.

AQUINO, A.D. Desidratação catalítica do etanol a éter etílico e etileno em alumina. Tese (mestrado). Campinas: Universidade Estadual de Campinas, Faculdade de Engenharia Química, 1989.

FOGLER, H.S. Elementos de Engenharia das Reações Químicas, 4 ed. Rio de Janeiro: Editora LTC, 2009.

KAGYRMANOVA, A.P.; CHUMACHENKO, V.A.; KOROTKIKH, V.N.; KASHKIN, V.N.; NOSKOV, A.S. Catalytic dehydration of bioethanol to ethylene: pilot-scale studies and process simulation. Chemical Engineering Journal, v.176, p.188-194, 2011.

KOLIOS, G.; FRAUHAMMER, J.; EIGENBERGER, G. Efficient reactor concepts for coupling of endothermic and exothermic reactions. Chemical Engineering Science, v.57, p.1505-1510, 2002.

LIMA, A.M. Estudo termodinâmico, cinético e otimização da produção de etileno a partir do etanol em alumina e óxido misto de cério-zircônio. Tese (mestrado). Uberlândia: Universidade Federal de Uberlândia, Faculdade de Engenharia Química, 2010.

LUTZE, P.; GANI, R.; WOODLEY, J.M. Process Intensification: A perspective on process synthesis. Chemical Engineering and Processing, v.49, p.547-558, 2010.

RAHIMPOUR, M.R.; DEHNAVI, M.R.; ALLAHGHOLIPOUR, F.; IRANSHAHI, D.; JOKAR, S.M. Assessment and comparison of different catalytic coupling exothermic and endothermic reactions: A review. Applied Energy, v.99, p.496-512, 2012.

ZANFIR, M.; GAVRIILIDIS, A. Modelling of a catalytic plate reactor for dehydrogenationcombustion coupling. Chemical Engineering Science, v.56, p.2671-2683, 2001. 